\title{
IDENTIFICAÇÃO E CLASSIFICAÇÃO DE ESTUDOS HISTÓRICOS CORRELATOS À ORGANIZAÇÃO CURRICULAR DE CURSOS DE MATEMÁTICA NO BRASIL
}

\author{
DESCRIBING AND CLASSIFICATION OF HISTORICAL STUDIES \\ RELATED TO THE ORGANIZATION OF MATHEMATICS COURSES IN \\ BRAZIL
}

\author{
Suélen Rita Andrade Machado ${ }^{1}$ \\ Lucieli Maria Trivizoli ${ }^{2}$
}

\begin{abstract}
Resumo: Neste artigo de revisão bibliográfica, identificamos e classificamos estudos relacionados à organização histórica e curricular de cursos de Matemática de instituições superiores no Brasil. A identificação dos trabalhos ocorreu no Catálogo de Teses e Dissertações do banco de dados da Coordenação de Aperfeiçoamento de Pessoal de Nível Superior (CAPES). Na primeira etapa selecionamos trabalhos relacionados à área da Matemática, e submetemos esses a uma pré-leitura ou leitura de reconhecimento com a finalidade de obter uma visão global do tema tratado. Escolhemos então, trabalhos correlatos e procuramos elencar seu objeto de estudo, objetivos, métodos e resultados. Posteriormente, classificamos os trabalhos nos campos investigativos em História da Matemática, História da Educação Matemática e História na Educação Matemática. Por fim, identificamos a relevância da classificação desses trabalhos, enquanto produto do conhecimento de práticas sociais investigativas relativas à História da Matemática, uma vez que reafirmam a autonomia desse campo de pesquisa.
\end{abstract}

Palavras-chave: Cursos de Matemática; Currículos dos Cursos de Matemática; História da Educação Matemática.

\begin{abstract}
In this bibliographical review, we identify and classify historical studies related to the historical and curricular organization of Mathematics courses of higher institutions in Brazil. The identification of researches published in the Catalog of Dissertations and Theses of the database of the Coordenação de Aperfeiçoamento de Pessoal de Nível Superior (CAPES). In the first step we selected researches related to the field of Mathematics and subjected them to a pre-reading or recognitional reading in order to get a global view of the theme. We then choose related researches and seek to list their object of study, objectives, methods and results. Subsequently, we classified the researches in the investigative fields of History of Mathematics, History of Mathematical Education and History in Mathematical Education. Finally, we understand the relevance of the identification and classification of these researches, as a product of knowledge of investigative social practices related to the History of Mathematics, while they reaffirm the autonomy of this field of research.
\end{abstract}

Keywords: Math courses; Mathematics Courses Curriculum; History of Mathematical Education.

\footnotetext{
${ }^{1}$ Mestre em Educação para a Ciência e a Matemática pela Universidade Estadual de Maringá (UEM). Professora da Faculdade de Engenharia e Inovação Técnico Profissional (FEITEP), Maringá, Paraná, Brasil. E-mail: sumachado18@gmail.com

${ }^{2}$ Doutora em Educação Matemática pela Universidade Estadual Paulista Júlio de Mesquita Filho - campus Rio Claro (UNESP). Professora Adjunta no Departamento de Matemática da Universidade Estadual de Maringá (UEM), Maringá, Paraná, Brasil. E-mail: $\underline{\text { Imtrivizoli@uem.br }}$
} 


\section{Introdução}

Este artigo pode ser incluído no conjunto de pesquisas historiográficas relacionadas à História da Educação Matemática Brasileira, cujo campo de pesquisa também se relaciona à História da Matemática, um campo relativamente recente no Brasil e que se entrelaça ao conhecimento Matemático, à Educação Matemática e à História das Ciências, por herdar, inevitavelmente, os métodos e técnicas atribuídos dessas áreas. Por sua vez, a História da Educação Matemática também dialoga com a História, a Educação e a Matemática recorrendo a diversas outras áreas do conhecimento, como filosofia, psicologia, antropologia, entre outras (TRIVIZOLI, 2016; GARNICA; SOUZA, 2012).

Temos ciência de que a história, em seu sentido mais abrangente, tem servido aos mais diversos propósitos da sociedade, como também a afirmação de identidades, a elucidação de práticas educativas e a difusão do conhecimento, como da Matemática, por exemplo. Os estudos dirigidos a essa área, fundamentados no reconhecimento e na interpretação de registros de fatos, datas, nomes e seus contextos, norteiam-se a partir da compreensão dos eventos e seus contextos e concepções. No caso da História da Matemática, a compreensão de seus eventos e contextos, bem como seu reconhecimento e interpretação, devem estar em concordância ao que se entende por Matemática (D`AMBROSIO, 1999).

Neste sentido, entendemos que a Matemática pode ser entendida como conjuntos de práticas sociais desenvolvidas para solução de problemas de ordens diversas (MIGUEL; MIORIM, 2002). Além disso, a própria História da Matemática é vista como uma possibilidade de vínculo à História da Educação Matemática e somos coniventes a um de seus propósitos de estudar “[...] como as comunidades se organizavam para produzir, usar e compartilhar conhecimentos matemáticos e como, afinal de contas, as práticas do passado podem - se é que podem - nos ajudar a compreender, projetar, propor e avaliar as práticas do presente" (GARNICA; SOUZA, 2012, p. 27).

Neste trabalho, apresentaremos como o título pressupõe, a identificação e classificação de trabalhos de pesquisas de cunho histórico correlatos à organização dos currículos de Cursos de Matemática no Brasil. Frisa-se que os trabalhos aqui relatados corroboram na construção de um panorama que, a princípio, deu suporte à constituição teórica, metodológica e analítica da dissertação defendida no âmbito do Programa de PósGraduação em Educação para a Ciência e a Matemática (PCM) da Universidade Estadual de Maringá (MACHADO, 2019). 
DOI: http://dx.doi.org/10.33238/ReBECEM.2019.v.3.n.3.23595

Ainda para fins deste trabalho, apresentaremos uma exposição dessas pesquisas relativa ao objetivo estabelecido para este escrito. Deste modo, entendemos a importância de situar essas pesquisas histórico-institucionais no contexto da História da Educação Matemática, já que não tratam somente de aspectos históricos, mas congregam, entre outros elementos, aspectos curriculares de Cursos de Matemática de instituições superiores do Brasil.

De acordo com Trivizoli (2009), o resgate histórico-institucional baseia-se no fato de que o fazer história não se reduz apenas ao olhar para o passado, mas a inclinação aos registros que evidenciam a origem da participação de pessoas dispostas em sociedade e envolvidas no processo de desenvolvimento científico, como também na organização e estruturação de cursos de Matemática de instituições superiores no Brasil. Ainda por essa pesquisadora, esse fazer História da Matemática, especificamente a História da Matemática no Brasil, é relativamente recente, já que os principais esforços, pesquisas e materiais produzidos nessa seara se intensificaram na década de 1990, principalmente pela realização de eventos específicos, como os Seminários Nacionais de História da Matemática. Neste sentido, a História da Matemática no Brasil é um elemento que possibilita a observação do desenvolvimento local da Matemática em relação aos centros científicos padrões, como também permite a compreensão da trajetória da Matemática no Brasil e seus expoentes, considerando acontecimentos, pessoas e registros.

Por se tratar de um estudo que se insere na abordagem de pesquisas qualitativas, (BOGDAN; BIKLEN, 1994), definimos como procedimentos metodológicos para este artigo, a revisão bibliográfica e a leitura do conteúdo em conformidade a Cervo, Bervian e Silva (2007) para identificação e interpretação das pesquisas. Para caracterização, definimos situar as pesquisas junto aos campos investigativos em História da Matemática situados por Miguel e Miorim (2002). Além dos campos definidos por esses autores, há outras pesquisas que analisaram produções acadêmicas em História da Matemática divulgadas em eventos da área e especificaram ramos de pesquisa nelas (SAD, 2005; MENDES, 2012).

Assumimos a priori que a revisão bibliográfica corrobora na aprendizagem de conhecimentos específicos, facilita a seleção e identificação de técnicas e métodos, e contribui na produção do corpo do trabalho científico. E, dentre as possibilidades de busca, inclui-se listas de citações de trabalhos recentes, publicações recentes e sumários de revistas da área, como também a busca pela rede mundial de computadores em bases de dados referenciais e textuais científicos, que apresenta em seu âmago, suporte para 


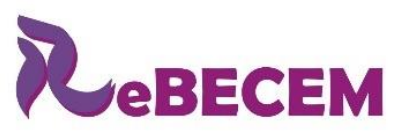

Revista Brasileira de Educação em

Ciências e Educação Matemática

DOI: http://dx.doi.org/10.33238/ReBECEM.2019.v.3.n.3.23595

busca de artigos e trabalhos científicos. As bases de dados listam referências bibliográficas de assuntos específicos incluindo o resumo, já as bases de dados textuais incluem outras informações numa base referencial, além de possibilitar o acesso ao arquivo (PIZZANI et al., 2012).

Definimos para esta investigação a base de dados textuais do Catálogo de Teses e Dissertações do banco de dados da Coordenação de Aperfeiçoamento de Pessoal de Nível Superior (CAPES), em busca de trabalhos relacionados às palavras-chave "Currículo do Curso" e "Curso de Matemática". Escolhemos tais palavras por ocasião da produção da dissertação que analisou os Currículos do Curso de Matemática de uma instituição de Ensino Superior no Estado do Paraná (MACHADO, 2019). À luz desse fato e da identificação prévia de trabalhos expressa na fase da Leitura Informativa ${ }^{3}$, realizamos uma Pré-Leitura e/ou Leitura de Reconhecimento que nos permitiu visualizar o objeto comum dos trabalhos e o tratamento dessas informações, com base na leitura dos títulos, resumos e palavras-chave (CERVO; BERVIAN; SILVA, 2007).

Posteriormente a esse reconhecimento, realizamos a Leitura Seletiva dos trabalhos, verificando similaridade em proximidade a nossa proposta investigativa, o que culminou na leitura reflexiva da dissertação de Valgas (2002), da tese de Ziccardi (2009), da tese de Castro e Almeida (2015) e da dissertação de Machado (2019) ${ }^{4}$ que apresentavam em comum o mesmo objeto de investigação, tratando de aspectos relativos à criação histórica, aspectos curriculares, legitimação e organização de Curso de Matemática de Instituições de Ensino Superior brasileiras. Para este artigo, realizamos a Leitura Interpretativa junto à etapa de Comentários do Texto, dimensionando os objetos de estudo, objetivos, métodos e resultados.

Nas próximas seções apresentaremos os trabalhos selecionados, posteriormente, trataremos dos campos investigativos em História da Matemática para classificar esses trabalhos. Finalmente, teceremos considerações sobre os estudos levantados e o campo investigativo da História da Matemática que se identificam como prática social investigativa.

\footnotetext{
${ }^{3}$ Levantamento e coleta de dados que dão respaldo a problematizações específicas, que engloba a Préleitura, Leitura Crítica ou Reflexiva, Leitura Interpretativa e Comentários de Texto (CERVO; BERVIAN; SILVA, 2007).

${ }^{4}$ A dissertação defendida por essa pesquisadora, ainda não se encontra na base de dados da CAPES por ter sido defendida recentemente, mas em virtude da correlação do objeto investigado com nosso objetivo, a identificamos no Repositório Institucional da Universidade Estadual de Maringá (RI-UEM).
} 


\section{Identificação e apresentação dos estudos}

Conforme Sacristán (2002), os estudos curriculares podem ser organizados e analisados sobre pontos de vista diversos, que incluem a função social curricular, da expressão formal do conteúdo e da estrutura, como campo prático que possibilita a análise da realidade e a interação da teoria e da prática, e as características que os tornam atividade discursiva acadêmica e de pesquisa sobre este tema.

Por considerar essas definições para caracterização de estudos curriculares, este artigo resultou de um levantamento de trabalhos para produção da dissertação realizada, que investigou os Currículos do Curso de Matemática de uma instituição de Ensino Superior no Estado do Paraná e que se insere nesta linha, como já elencamos. Cientes disto, fizemos um levantamento no catálogo do banco de dados textual da CAPES a partir de palavras-chave específicas, a fim de refinar trabalhos comuns ao nosso objeto. Especificamos para busca, as palavras-chave: "Currículo do Curso" e "Curso de Matemática".

Para a palavra-chave "Currículo do Curso", considerando a abrangência deste termo, realizamos ainda, um refinamento na busca por trabalhos conexos ao campo da Matemática, e então encontramos os trabalhos de Valgas (2002) e Lima (2012). Já para a palavra-chave "Curso de Matemática", encontramos os trabalhos de Bortoli (2003), Ziccardi (2009), Lima (2012), Santos (2013), Soares (2013), Castro e Almeida (2015) e Araujo Neto (2016).

Além dos trabalhos identificados, consideramos também a pesquisa de Machado (2019) que ainda não está disponível no banco de dados da $\mathrm{CAPES}^{5}$. Entendemos que ela deve se fazer presente no processo de identificação de estudos curriculares sobre cursos de Matemática no Brasil, dada a sua abordagem em relação à análise das modificações curriculares de um curso de Matemática do interior do Paraná.

Assim, para apresentação e visualização das informações, ordenamos os dados no quadro 1, de modo a facilitar a leitura dos trabalhos no que se refere às palavras-chave, modalidade de trabalho, nome dos autores, ano de publicação e nome do orientador.

\footnotetext{
${ }^{5}$ Esse trabalho foi defendido no dia 25/02/2019 e se encontra no Repositório Institucional da Universidade Estadual de Maringá.
} 


\begin{tabular}{|c|c|c|}
\hline \multicolumn{3}{|c|}{ 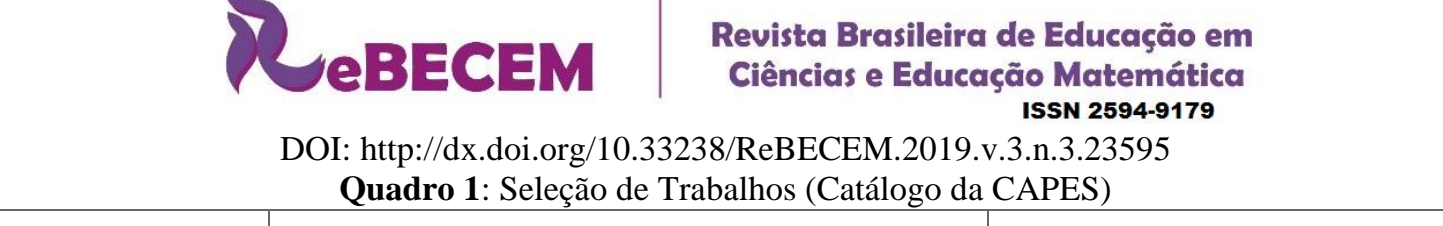 } \\
\hline Palavras-chave & Trabalho/Modalidade & $\begin{array}{c}\text { Autor(a)/Ano de } \\
\text { Publicação/Orientador(a) }\end{array}$ \\
\hline & $\begin{array}{l}\text { Licenciatura em Matemática: aspectos históricos } \\
\text { e curriculares da UEPG. (Mestrado) }\end{array}$ & $\begin{array}{l}\text { Carmen Lúcia Valgas (2002) } \\
\text { Or (a): Teresa Jussara Luporini }\end{array}$ \\
\hline $\begin{array}{l}\text { Currículo do } \\
\text { Curso }\end{array}$ & $\begin{array}{c}\text { A disciplina de Cálculo I do curso de } \\
\text { Matemática da Universidade de São Paulo: um } \\
\text { estudo de seu desenvolvimento, de } 1934 \text { a } 1994 . \\
\text { (Doutorado) }\end{array}$ & $\begin{array}{c}\text { Gabriel Loureiro de Lima } \\
\text { (2012) } \\
\text { Or: Benedito Antonio da Silva }\end{array}$ \\
\hline \multirow{8}{*}{$\begin{array}{l}\text { Curso de } \\
\text { Matemática }\end{array}$} & $\begin{array}{l}\text { História da Criação do Curso de Matemática na } \\
\text { Pontifícia Universidade Católica de Campinas. } \\
\text { (Mestrado) }\end{array}$ & $\begin{array}{l}\text { Adriana de Bortoloni (2003) } \\
\text { Or: Marcos Vieira Teixeira }\end{array}$ \\
\hline & $\begin{array}{l}\text { O curso de Matemática da PUC/SP: uma história } \\
\text { de sua construção/desenvolvimento/legitimação. } \\
\text { (Doutorado) }\end{array}$ & $\begin{array}{l}\text { Lydia Rossana Nocchi } \\
\text { Ziccardi (2009) } \\
\text { Or: Ubiratan D'Ambrosio }\end{array}$ \\
\hline & $\begin{array}{c}\text { A disciplina de Cálculo I do curso de } \\
\text { Matemática da Universidade de São Paulo: um } \\
\text { estudo de seu desenvolvimento, de } 1934 \text { a } 1994 . \\
\text { (Doutorado) }\end{array}$ & $\begin{array}{c}\text { Gabriel Loureiro de Lima } \\
(2012) \\
\text { Or: Benedito Antonio da Silva }\end{array}$ \\
\hline & $\begin{array}{l}\text { Formação Superior Específica de Professores de } \\
\text { Matemática no Espírito Santo: Uma História de } \\
\qquad 1964 \text { a 2000. (Mestrado) }\end{array}$ & $\begin{array}{l}\text { Marina Gomes dos } \\
\text { Santos (2013) } \\
\text { Or (a): Lígia Arantes Sad }\end{array}$ \\
\hline & $\begin{array}{l}\text { Um estudo histórico do ensino de geometria } \\
\text { analítica no curso de matemática da UFJF nas } \\
\text { décadas de } 1960 \text { e } 1970 . \\
\text { (Mestrado Profissional) }\end{array}$ & $\begin{array}{l}\text { Susana Ribeiro Soares (2013) } \\
\text { Or (a): Maria Cristina Araújo } \\
\text { de Oliveira }\end{array}$ \\
\hline & $\begin{array}{l}\text { Um lugar: muitas histórias - o processo de } \\
\text { formação de professores de Matemática } \\
\text { na primeira instituição de ensino superior da } \\
\text { região de Montes Claros/ norte de Minas } \\
\text { Gerais (1960-1990) }\end{array}$ & $\begin{array}{l}\text { Shirley Patrícia Nogueira de } \\
\text { Castro e Almeida } \\
\text { (2015) } \\
\text { Or (a): Maria Laura Magalhães } \\
\text { Gomes }\end{array}$ \\
\hline & $\begin{array}{l}\text { Um Estudo Histórico do Curso de Matemática } \\
\text { da Universidade Estadual de Maringá: A criação } \\
\text { e os primeiros anos. (Mestrado) }\end{array}$ & $\begin{array}{l}\text { Antonio Peixoto de Araujo } \\
\text { Neto (2016) } \\
\text { Or (a): Lucieli M. Trivizoli }\end{array}$ \\
\hline & $\begin{array}{l}\text { As modificações curriculares do Curso de } \\
\text { Matemática da Universidade Estadual de } \\
\text { Maringá: Mudanças no saber profissional do } \\
\text { professor de Matemática, 1971-1996. } \\
\text { (Mestrado) }\end{array}$ & $\begin{array}{c}\text { Suélen Rita Andrade Machado } \\
\text { (2019) } \\
\text { Or (a): Lucieli M. Trivizoli }\end{array}$ \\
\hline
\end{tabular}

Fonte: Autoras

Dos resultados das pesquisas encontradas para a palavra-chave "Currículo do Curso", definimos para esta exposição o trabalho de Valgas (2002) e omitimos o trabalho de Lima (2012) por se caracterizar como um estudo sobre o desenvolvimento da disciplina de Cálculo I do curso de Matemática da Universidade de São Paulo de 1934 a 1994. 
DOI: http://dx.doi.org/10.33238/ReBECEM.2019.v.3.n.3.23595

Ressaltamos, que embora essa pesquisa congregue um estudo histórico e curricular, se limita a análise de uma disciplina.

Dos resultados de pesquisas encontradas para a palavra-chave "Curso de Matemática”, elegemos o trabalho de Ziccardi (2009) e Castro e Almeida (2015) omitimos os demais trabalhos, em virtude de que tanto o trabalho de Lima (2012) quanto o de Soares (2013), tratam de estudos históricos e curriculares referentes à duas disciplinas do currículo de Cursos de Matemática de instituições superiores no Brasil, como a disciplina de Cálculo I na USP e a disciplina de Geometria Analítica na UFJF, respectivamente.

Já as pesquisas de Bortoli (2003) e Araujo Neto (2016) trataram da criação e dos primeiros anos de Cursos de Matemática de instituições superiores; quanto ao trabalho de Santos (2013), trata-se de um estudo acerca da constituição histórica de cursos relacionados a formação superior específica de professores de Matemática no estado do Espírito Santo de 1964 a 2000, tomando como recorte a criação do curso de Matemática da Faculdade de Filosofia, Ciências e Letras do Espírito Santo, em 1964.

Do processo de identificação de trabalhos, obtemos, então, a dissertação de Valgas (2002) que se refere à palavra-chave "Currículo do Curso", a tese de Ziccardi (2009) e Castro e Almeida (2015) que se refere a palavra-chave "Curso de Matemática". Como já elencamos, consideraremos a dissertação de Machado (2019), que entendemos se inserir nas duas modalidades de palavras-chave. Por conseguinte, tendo em vista as justificativas anteriormente apresentadas, limitamos essa identificação a esses quatro respectivos trabalhos, uma vez que são estudos históricos correlatos a organização de cursos e currículos de Cursos de Matemática de instituições superiores no Brasil, em localidades distintas e que se baseiam na historiografia e na análise documental de modo a cumprir seu objetivo. Nesse sentido, na sequência apresentamos um breve resumo sobre o conteúdo dessas pesquisas, possíveis aproximações e divergências entre elas.

Apresentamos primeiramente a pesquisa de mestrado de Valgas (2002) que apresenta como título Licenciatura em Matemática: Aspectos Históricos e Curriculares na UEPG. Esta pesquisa trata sobre as principais mudanças ocorridas no currículo do Curso de Licenciatura em Matemática da Universidade Estadual de Ponta Grossa UEPG, Paraná, a partir da trajetória histórica do curso, considerando as Faculdades de Ciências e Letras com seus cursos, que foram reunidas para compor esta universidade.

A investigação envolveu um estudo histórico das principais reformulações das grades curriculares desde a criação do Curso de Matemática, de 1950 até o ano de 2002, 
DOI: http://dx.doi.org/10.33238/ReBECEM.2019.v.3.n.3.23595

por meio da análise documental de arquivos físicos, como atas, grades curriculares, resoluções e entrevistas com docentes que atuaram no Departamento de Matemática daquela instituição. Por meio de um estudo exploratório-descritivo, a pesquisadora evidenciou três reformulações importantes ao longo do histórico do curso, que segundo a autora se limitou a alteração de carga horária de disciplinas e nomenclaturas destas, apenas a última alteração referente ao ano de 1996, é considerada pela pesquisadora como uma reformulação profunda, uma vez que mudou a visão do curso referente a concepção de licenciatura propagada pelo curso (VALGAS, 2002).

Já a pesquisa de doutorado de Ziccardi (2012) apresenta como título $O$ curso de Matemática da Pontifícia Universidade Católica de São Paulo: uma história de sua construção/desenvolvimento/legitimação. Ao tomar como objeto o Curso de Matemática da Pontifícia Universidade Católica de São Paulo - PUC-SP, a pesquisadora apresentou a trajetória e a criação da PUC-SP, como também a criação, consolidação e legitimação do Curso de Matemática nessa instituição, a partir da organização e estrutura acadêmica, constituição docente e discente, como também a constituição histórica da Pós-graduação em Matemática.

Ziccardi (2012) apresentou e analisou as grades curriculares que configuraram o curso, a participação de professores na constituição desses currículos e a importância de determinadas pessoas do curso ligadas ao ensino e à pesquisa, que corroboraram no campo da Educação Matemática Brasileira. Em relação a análise das reformulações curriculares do Curso de Matemática, a pesquisadora evidenciou por meio da análise de documentos físicos e entrevistas, que alterações curriculares podem ser constadas a partir do ano de 1982, quando os professores do curso se preocupavam com participação dos alunos em atividades extracurriculares, como também no ano de 1995, quando o curso separou a modalidade licenciatura e bacharelado, visto que até aquele momento quem se formava em licenciatura no curso também se formava em bacharel, contudo, no ano 2000 , o curso de Matemática se estabelece como estritamente licenciatura, na qual começa a se pensar e colocar em prática, reformulações integradas às tendências emergidas no campo da Educação Matemática (ZICCARDI, 2009).

A pesquisa de doutorado de Castro e Almeida (2015) intitulada Um lugar: muitas histórias - o processo de formação de professores de Matemática na primeira instituição de ensino superior da região de Montes Claros/ norte de Minas Gerais (1960-1990), constatou que o primeiro curso de Matemática daquela região, foi criado em 1968 e foi subsidiado por uma instituição privada da região, nomeada Fundação Educacional Luiz 
DOI: http://dx.doi.org/10.33238/ReBECEM.2019.v.3.n.3.23595

de Paula (FELP) e, posteriormente, incorporado à Fundação Norte Mineira de Ensino Superior (FUNM), que passou sob autarquia estadual a ser denominada em 1990, Universidade Estadual de Montes Claros - UNIMONTES.

O curso de matemática referido anteriormente, foi constituído e organizado sob as influências políticas, econômicas e educacionais vigentes à época. Destaca-se segundo a pesquisadora, um currículo fomentando por estudos dos conteúdos de disciplinas matemáticas e disciplinas pedagógicas.

Por fim, a pesquisa de mestrado de Machado (2019) intitulada As modificações curriculares do Curso de Matemática da Universidade Estadual de Maringá: mudanças no saber profissional do professor de matemática, 1971-1996, apresenta a partir de aspectos históricos e legislativos, uma revisão acerca dos primeiros anos do Curso de Matemática da Universidade Estadual de Maringá, com base no estudo histórico de Araujo Neto (2016) e então, apresentou as modificações curriculares do Curso de Matemática da UEM, dos anos 1971 a 1996, a partir da análise documental de arquivos inventariados recentemente do Departamento de Matemática da UEM (MACHADO; TRIVIZOLI, 2019).

A partir da análise dos documentos acumulados ao longo de vinte cinco anos, Machado (2019) evidenciou quatro currículos para o Curso de Matemática, e alterações no que se refere a rubricas e inserções/exclusões de disciplinas do rol curricular. Quanto ao saber curricular advindos dos currículos constatados, concluiu que o curso oferecia disciplinas de conteúdo matemático e disciplinas pedagógicas, consoantes aos aspectos legislativos daquele momento histórico.

Em relação às aproximações entre os trabalhos, verificamos que os trabalhos escolhidos podem ser amparados na historiografia brasileira que envolve desde o Histórico da Educação Matemática no Brasil, o estudo curricular e o históricoinstitucional de instituições superiores situadas em distintas localidades brasileiras, duas no Estado do Paraná, outra no Estado de São Paulo e outra no Estado de Minas Gerais. Ambas se primam na análise de modificações curriculares de Cursos de Matemática das instituições superiores que o inserem, com base na análise documental de arquivos físicos e históricos. Ressaltam em comum as principais reformulações relacionadas aos aspectos legislativos e voltadas a licenciatura na dimensão que assentam suas investigações e no caso específico de suas instituições.

Ainda, os trabalhos analisados tratam de duas pesquisas de mestrado e duas de doutorado. Visto que, Valgas (2002) escreveu sua dissertação baseada na teoria curricular 
DOI: http://dx.doi.org/10.33238/ReBECEM.2019.v.3.n.3.23595

e nos conceitos atrelados ao currículo, posteriormente apresentou o histórico da UEPG, inseriu o Curso de Matemática nesse leque e tratou das reformulações mediante a legislação, a análise documental e as entrevistas semiestruturadas dirigidas aos docentes do Departamento afeto. Não se debruçou a situar o campo da Educação Matemática e suas contribuições no currículo do curso como fez Ziccardi (2009) e Machado (2019). Frisa-se que Machado (2019) lançou suas bases estritamente na pesquisa documental e analisou a as modificações curriculares do Curso de Matemática da UEM no que se refere principalmente aos aspectos legislativos e aos saberes profissionais do professor de matemática em formação naquele período histórico.

Outro fato, diz respeito a UEPG e a UEM serem consideradas instituições estaduais com características e filosofias atreladas às condições políticas, econômicas e sociais da localidade que se insere. Já, a pesquisa de Ziccardi (2009) por se tratar de uma pesquisa que toma como objeto a instituição PUC-SP, mostra em seu escrito o histórico de uma instituição privada, com uma filosofia religiosa que permite a autonomia de pesquisa e sua preocupação com o ensino e aprendizagem contextualizado com as tendências do momento. Seu escrito mobiliza a ideia de uma instituição expressa em independência, pelas condições de uma universidade formada por um conjunto de faculdades isoladas e independentes, que se baseavam em uma ideologia religiosa católica, que fomentava a pesquisa e não se eximia ao governo. Já a pesquisa de Castro e Almeida (2015) tratou de uma instituição que até o recorte histórico de análise era concebida como privada, vindo a se tornar em 1990 uma instituição estadual.

Nos quadros 2 e 3 apresentamos a identificação que definimos para a seleção dos quatro trabalhos, em relação ao seu objeto, objetivo geral, metodologia e resultados principais. Como a descrição das categorias de descrição são densas, foram elaborados dois quadros, apresentando na parte I e na parte II, dois trabalhos respectivamente:

Quadro 2: Identificação dos Trabalhos (Ilustrativa) - Parte I

\begin{tabular}{|c|c|c|}
\hline $\begin{array}{l}\text { Trabalhos } \\
\text { Selecionados }\end{array}$ & $\begin{array}{l}\text { Licenciatura em Matemática: } \\
\text { Aspectos Históricos e } \\
\text { Curriculares na UEPG } \\
\text { (VALGAS, 2002) }\end{array}$ & $\begin{array}{c}\text { O Curso de Matemática da Pontifícia } \\
\text { Universidade Católica de São Paulo: uma } \\
\text { história de sua } \\
\text { construção/desenvolvimento/legitimação } \\
\text { (ZICCARDI, 2009) }\end{array}$ \\
\hline \multirow[t]{2}{*}{ Objeto } & $\begin{array}{c}\text { Mudanças Curriculares do Curso } \\
\text { de Matemática da UEPG. }\end{array}$ & $\begin{array}{l}\text { O Curso de Matemática da PUC-SP: a } \\
\text { trajetória do curso, sua organização e estrutura } \\
\text { acadêmica, suas realizações, } \\
\text { seus planos e projetos que envolveram seus } \\
\text { dirigentes, professores e alunos. }\end{array}$ \\
\hline & $\begin{array}{c}\text { Conhecer a trajetória histórica do } \\
\text { Curso de Licenciatura em } \\
\text { Matemática da UEPG para }\end{array}$ & Investigar o processo de criação e \\
\hline
\end{tabular}


DOI: http://dx.doi.org/10.33238/ReBECEM.2019.v.3.n.3.23595

\begin{tabular}{|c|c|c|}
\hline Objetivo Geral & $\begin{array}{l}\text { compreender o alcance e } \\
\text { significado de suas principais } \\
\text { reformulações curriculares. }\end{array}$ & $\begin{array}{l}\text { legitimação do Curso de Matemática da } \\
\text { Pontifícia Universidade Católica de São } \\
\text { Paulo, Bacharelado e Licenciatura. }\end{array}$ \\
\hline Metodologia & $\begin{array}{l}\text { Pesquisa Exploratório-Descritiva } \\
\text { que envolveu pesquisa } \\
\text { bibliográfica e documental, } \\
\text { Análise Documental e Entrevistas } \\
\text { Semiestruturadas. }\end{array}$ & $\begin{array}{l}\text { Pesquisa historiográfica, baseada em pesquisa } \\
\text { documental com } \\
\text { fontes primárias e secundárias, obras de } \\
\text { referência e na análise de } \\
\text { entrevistas. }\end{array}$ \\
\hline Resultados & $\begin{array}{l}\text { Foi detectado que desde a criação } \\
\text { do curso de Licenciatura em } \\
\text { Matemática em } 1950 \text { até o ano de } \\
\text { 2002, as mudanças ocorridas no } \\
\text { currículo foram apenas } \\
\text { relacionadas em sua maioria a } \\
\text { alteração de carga horária de } \\
\text { disciplinas, nomenclatura de } \\
\text { disciplinas, aspectos legislativos, } \\
\text { entre outros. } \\
\text { Do período que compreendeu a } \\
\text { investigação histórica, a } \\
\text { pesquisadora definiu três } \\
\text { modificações curriculares } \\
\text { consideradas significativas para } \\
\text { estruturação do curso, a de 1975, } \\
\text { quando o curso passa de } \\
\text { Licenciatura em Matemática para } \\
\text { Licenciatura em Ciências; a de } \\
\text { 1985, quando o curso volta a ser } \\
\text { Licenciatura em Matemática; por } \\
\text { fim, a de 1996, considerada pela } \\
\text { pesquisadora como uma } \\
\text { reformulação profunda, uma vez } \\
\text { que mudou a visão do curso e a } \\
\text { concepção de licenciatura. }\end{array}$ & 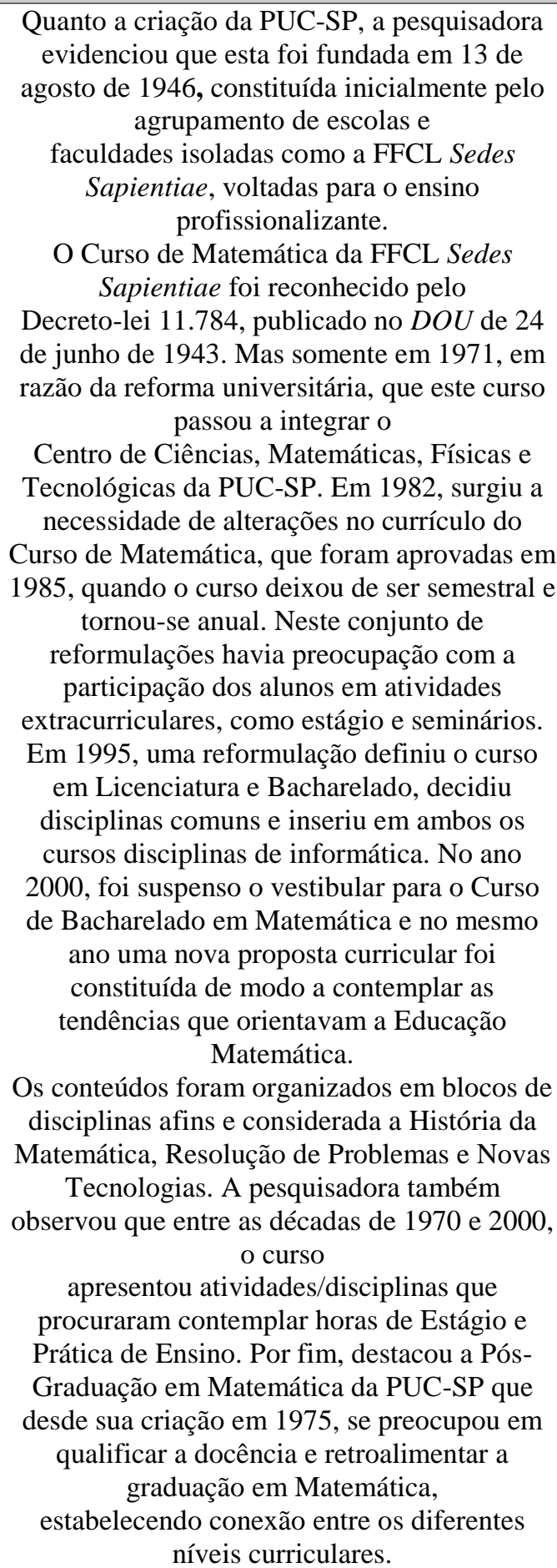 \\
\hline
\end{tabular}

Fonte: Autoras 
DOI: http://dx.doi.org/10.33238/ReBECEM.2019.v.3.n.3.23595

Quadro 3: Identificação dos Trabalhos (Ilustrativa) - Parte II

\begin{tabular}{|c|c|c|}
\hline $\begin{array}{c}\text { Trabalhos } \\
\text { Selecionados }\end{array}$ & $\begin{array}{l}\text { Um lugar: muitas histórias - o } \\
\text { processo de formação de } \\
\text { professores de Matemática } \\
\text { na primeira instituição de ensino } \\
\text { superior da região de Montes } \\
\text { Claros/ norte de Minas } \\
\text { Gerais (1960-1990) } \\
\text { (CASTRO E ALMEIDA, 2015) }\end{array}$ & $\begin{array}{l}\text { As modificações curriculares do Curso de } \\
\text { Matemática da Universidade Estadual de } \\
\text { Maringá: mudanças no saber profissional } \\
\text { do professor de matemática, 1971-1996 } \\
\text { (MACHADO, 2019) }\end{array}$ \\
\hline Objeto & $\begin{array}{l}\text { O processo formativo de } \\
\text { professores de Matemática na } \\
\text { região norte de Monte Claros. }\end{array}$ & $\begin{array}{l}\text { As modificações curriculares do Curso de } \\
\text { Matemática da UEM. }\end{array}$ \\
\hline Objetivo Geral & $\begin{array}{l}\text { Investigar o processo de formação } \\
\text { de professores de Matemática na } \\
\text { primeira instituição de ensino } \\
\text { superior da região de Montes } \\
\text { Claros, Norte de Minas Gerais, no } \\
\text { período de } 1960 \text { a } 1990\end{array}$ & $\begin{array}{c}\text { Analisar as transformações curriculares do } \\
\text { Curso de Matemática de modo } \\
\text { a caracterizar as mudanças do saber } \\
\text { profissional do professor de Matemática da } \\
\text { UEM. }\end{array}$ \\
\hline Metodologia & $\begin{array}{c}\text { Pesquisa documental e História } \\
\text { Oral. }\end{array}$ & $\begin{array}{c}\text { Pesquisa Exploratório-Descritiva, baseada na } \\
\text { pesquisa documental em } \\
\text { fontes primárias e secundárias. }\end{array}$ \\
\hline Resultados & $\begin{array}{l}\text { O primeiro curso de Matemática } \\
\text { daquela região, foi criado em } 1968 \\
\text { e foi subsidiado por uma instituição } \\
\text { privada da região, nomeada } \\
\text { Fundação Educacional Luiz de } \\
\text { Paula (FELP) e, posteriormente, } \\
\text { incorporado à Fundação Norte } \\
\text { Mineira de Ensino Superior } \\
\text { (FUNM), que passou sob autarquia } \\
\text { estadual a ser denominada em 1990, } \\
\text { Universidade Estadual de Montes } \\
\text { Claros - UNIMONTES. } \\
\text { O curso em si, foi constituído e } \\
\text { organizado sob influências de bases } \\
\text { políticas, econômicas e } \\
\text { educacionais do cenário que residia } \\
\text { o momento histórico. } \\
\text { A formação do curso mantinha } \\
\text { aspectos similares com outros } \\
\text { cursos a nível nacional, } \\
\text { considerando as políticas } \\
\text { educacionais do momento. } \\
\text { Destaca-se segundo a pesquisadora, } \\
\text { um currículo fomentando por } \\
\text { estudos dos conteúdos de } \\
\text { disciplinas matemáticas e } \\
\text { disciplinas pedagógicas. }\end{array}$ & $\begin{array}{l}\text { Constatou que o modelo curricular assumido } \\
\text { pelo Curso de Matemática da Instituição } \\
\text { UEM, desde a sua criação até 1996, se } \\
\text { assemelha a outros cursos de Licenciatura em } \\
\text { Matemática, mantidos em outras instituições } \\
\text { a nível nacional, conforme o modelo } 3+1 \text { de } \\
\text { formação da época, que concebia uma } \\
\text { formação voltada em sua maioria aos } \\
\text { conceitos matemáticos e uma mínima parte } \\
\text { para os conceitos pedagógicos. } \\
\text { Desse modo, verificou nas documentações } \\
\text { quatro diferentes currículos e suas variações } \\
\text { ao longo de } 25 \text { anos de existência do curso. } \\
\text { Destaca-se, segundo a pesquisadora, a } \\
\text { inserção curricular de disciplinas } \\
\text { relacionadas a instrumentalização do ensino } \\
\text { da matemática, a partir da década de 1980, } \\
\text { com a finalidade de instrumentalizar a } \\
\text { formação de professores de matemática para } \\
\text { o Ensino Secundário. } \\
\text { Ainda nesse mesmo período, parte do corpo } \\
\text { docente do curso aderiu as primeiras } \\
\text { iniciativas para o movimento da Educação } \\
\text { Matemática no Brasil. } \\
\text { Entretanto, em } 1996 \text { o curso passou a } \\
\text { oferecer a modalidade Licenciatura e } \\
\text { Bacharelado. }\end{array}$ \\
\hline
\end{tabular}




\section{Campos investigativos em história da matemática: classificação dos estudos}

\section{identificados}

Escolhemos nesta seção tratar dos campos investigativos em História da Matemática definidos por Miguel e Miorim (2002), pois representam a visão desses pesquisadores face ao objeto da produção acadêmica no interior da prática social de investigação em História da Matemática. Com base na leitura específica de produção social em História da Matemática, esses autores definiram especificamente os campos: (C1) História da Matemática; (C2) História da Educação Matemática; (C3) História na Educação Matemática; (C4) Estudos Historiográficos; (C5) Teoria da História na ou da Educação Matemática; e (C6) Campos Afins.

Antecedente a essa categorização, os campos afins e investigativos da História da Matemática, da Educação Matemática e da História e Educação Matemática, já haviam sido apresentados por Miorim e Miguel (2001), como campos autônomos de investigação que vieram a se constituir no século $\mathrm{XX}$, com base nos primórdios históricos de seu desenvolvimento que indicam que esses campos se encontravam indissociados do campo da matemática; também, pela constituição de sua autonomia identificada no desenvolvimento histórico desses campos e baseada na publicação de trabalhos específicos da área, discussões coletivas e formação de sociedades e comunidades revelando preocupação, o desenvolvimento de investigações e o fortalecimento do novo campo do conhecimento.

Ainda por esses pesquisadores, foi possível verificar a existência do processo de constituição da autonomia desses campos, como também negar a relação de dependência entre eles, quando sobretudo a História da Matemática passou a ser contemplada pela educação matemática como um campo emergente ao diálogo, que viabiliza o desdobramento do campo da História da Matemática,

[...] nesse sentido, fazer 'história da matemática' passa a significar coisas distintas, tais como: fazer história da matemática propriamente dita, fazer história da educação matemática, realizar investigações teóricas ou de campo a respeito das relações entre história da matemática e educação matemática ou ainda fazer a história de todas essas histórias (MIORIM; MIGUEL, 2001, p. $60)$.

Com base na classificação de trabalhos científicos, Miguel e Miorim (2002) estabeleceram então seis campos investigativos sobre a prática social investigativa em História da Matemática, a qual apresentamos em síntese no enredo desta seção: 


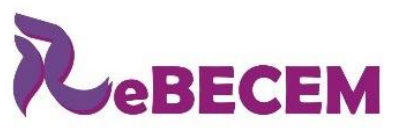

Revista Brasileira de Educação em

Ciências e Educação Matemática

DOI: http://dx.doi.org/10.33238/ReBECEM.2019.v.3.n.3.23595

O campo (C1) é concebido como um processo ou atividade complexa, que não se reduz ao estudo das ideias matemáticas pelo espaço temporal, mas se debruça ao "[...] estudo de natureza histórica que investiga, diacrônica ou sincronicamente, todas as dimensões de matemática na história em todas as práticas sociais que participam e/ou participaram do processo de produção de conhecimento matemático [...]” (MIGUEL; MIORIM, 2002, p. 186). Em síntese, os estudos nesse campo se preocupam com o processo de constituição dos produtos da atividade matemática, resultantes direta e/ou indiretamente de práticas sociais (TRIVIZOLI, 2016).

O campo (C2) também é concebido como um processo ou atividade complexa, que vai além das doutrinas educacionais e ideias pedagógicas ligadas à matemática, apresenta características similares ao campo anterior, em relação ao estudo das dimensões históricas da produção do conhecimento matemático por meio das práticas sociais, entretanto, se debruça “[...] a atividade matemática na história, exclusivamente em suas manifestações em práticas pedagógicas de circulação e apropriação do conhecimento matemático [...]" (MIGUEL; MIORIM, 2002, p. 187). Conforme Trivizoli (2016), um exemplo de fontes de estudo para esse campo, além da história de decretos educacionais, normas e currículos, são os estudos relativos a cadernos de alunos, memórias, cartas, livros didáticos entre outros.

O campo (C3), por sua vez, toma como objeto investigativo as formas de ação pedagógica que incluem a participação da História da Matemática no bojo escolar e seu tratamento em pesquisas que apontam ações docentes, discentes em relação ao enfoque histórico. O campo (C4) se constitui de estudos de natureza histórica e/ou filosófica que tomam como foco a Historiografia da Matemática ou da Educação Matemática (MIGUEL; MIORIM, 2002). Já o campo (C5) constitui-se de estudos de natureza filosófica voltados aos

[...] aspectos relativos à história na educação matemática e/ou à história da educação matemática, particularmente aqueles que se apresentam em fontes escritas diversas (documentos legais, livros didáticos, artigos relativos ao campo em questão, dissertações e teses, etc.) destinadas a quaisquer níveis de ensino e/ou em fontes orais (MIGUEL; MIORIM, 2002, p. 188).

Por fim, o campo (C6) é destinado para aqueles trabalhos que não se incluem nos demais campos classificados por Miguel e Miorim (2002).

Em relação aos trabalhos que apresentamos neste escrito mediante a síntese apresentada sobre cada campo, entendemos que tanto as dissertações de Valgas (2002) e Machado (2019) quanto as teses de Ziccardi (2009) e Castro e Almeida (2015) são estudos 


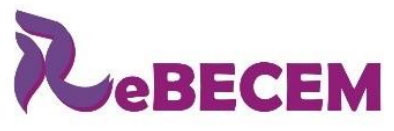

DOI: http://dx.doi.org/10.33238/ReBECEM.2019.v.3.n.3.23595
Revista Brasileira de Educação em

Ciências e Educação Matemática

ISSN 2594-9179

que correspondem ao campo (C2) História da Educação Matemática, uma vez que versam sobre o histórico-institucional de cursos de matemática de instituições de Ensino Superior, sobre a organização, estrutura e composição, as modificações curriculares e aspectos que indicam a representatividade do corpo docente desses cursos para o desenvolvimento histórico e curricular desses cursos.

Entretanto, entendemos que ambos os trabalhos podem se classificar em mais de um campo investigativo, uma vez que uma pesquisa pode envolver diversos campos que não se amortizam a apenas uma perspectiva enquanto prática social investigativa, nesse caso da História da Matemática. Desse modo, verificamos que os trabalhos acima listados se classificam também no campo (C4) Estudos Historiográficos, visto que manifestam em seu enredo um tratamento à Historiografia da Matemática e da Educação Matemática e se definem também como pesquisas historiográficas, como podemos observar em Ziccardi (2009), Castro e Almeida (2015) e Machado (2019).

Consideramos que os trabalhos identificados e classificados nos forneceram diversos panoramas, a saber como: dimensões metodológicas e analíticas em relação ao tratamento de fontes primárias e sua análise, os tipos de pesquisa que exploram e descrevem um problema; a estrutura organizacional seguida por estas pesquisas referentes ao encadeamento de informações históricas elencadas, a bibliografia referenciada, outras pesquisas identificadas no corpo do texto e relacionadas ao objeto de estudo, aspectos legislativos referentes ao currículo, aporte teórico para revisão bibliográfica referente ao ensino da matemática no Brasil desde os primórdios ao Ensino Superior, aspectos que caracterizam definições sobre currículos; como também a explanação dos resultados evidenciados nas pesquisas.

\section{Considerações}

Nesse trabalho tivemos como objetivo identificar e classificar estudos históricos relacionados à organização histórica e curricular de cursos de Matemática de instituições superiores no Brasil, com base em levantamento bibliográfico.

Do processo de identificação de trabalhos na base de dados textuais por meio de palavras-chave específicas, além dos trabalhos que apresentamos brevemente no conjunto deste texto, destacamos os trabalhos de Valgas (2002), Ziccardi (2009), Castro e Almeida (2015) e Machado (2019) que apresentaram em convergência como já mencionamos, o mesmo objeto de investigação, tratando de aspectos relativos a criação histórica, aspectos 
DOI: http://dx.doi.org/10.33238/ReBECEM.2019.v.3.n.3.23595

curriculares, legitimação e organização de Curso de Matemática de Instituições de Ensino Superior brasileiras.

No texto, procuramos descrever as aproximações e divergências entre cada trabalho, na qual tanto o trabalho de Ziccardi (2009) quanto o trabalho de Machado (2019) evidenciam a preocupação da reformulação curricular do curso de Matemática da PUCSP e da UEM em relação aos estudos da Educação Matemática, diferentemente do trabalho de Valgas (2002) e Castro e Almeida (2015) que apresentam reformulações relativas a prática da licenciatura.

Os trabalhos de Valgas (2002), Castro e Almeida (2015) e Machado (2019) apresentam como especificidade o tratamento das modificações curriculares de cursos de Matemática de instituições de nível superior no Brasil, inclinando na trajetória históricacurricular do curso, a partir de recortes temporais. Valgas (2002) e Machado (2019) concentram seus estudos para a UEPG e UEM, localizadas no interior Estado do Paraná, já Castro e Almeida (2015) trata da UNIMONTES, localizada no interior do Estado de Minas Gerais.

Já a tese de Ziccardi (2009) apresenta os componentes que suscitaram na organização e legitimação do curso de Matemática da Pontifícia Universidade Católica de São Paulo (PUC-SP), seu histórico, as grades curriculares, suas modificações, a representatividade dos professores na constituição dos currículos e a relação desses com o desenvolvimento da pesquisa e do ensino no âmbito da Educação Matemática.

Em relação ao processo de classificação destes trabalhos a partir dos campos investigativos em História da Matemática definidos por Miguel e Miorim (2002), entendemos sua relevância em possibilitar o conhecimento de práticas sociais investigativas relativas a História da Matemática, uma vez que reafirmam a autonomia desses campos de pesquisa. E podemos considerar, ainda, que o presente artigo pode ser inserido, também, nos campos da História da Educação Matemática e Estudos Historiográficos.

A par dos campos de pesquisa, constatamos que não somente os quatro trabalhos selecionados, mas também os trabalhos identificados no quadro 1, se configuram como estudos relativos ao Campo da História da Educação Matemática, por tratar em seu enredo sobre manifestações pedagógicas, pertinentes a história em termos locais da Matemática no Brasil. Entretanto, por tratarem em seu enredo sobre aspectos historiográficos da História da Educação Matemática, consideramos que esses trabalhos também se aplicam ao Campo de Estudos Historiográficos. 


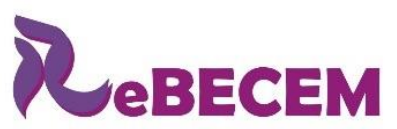

Revista Brasileira de Educação em

Ciências e Educação Matemática

ISSN 2594-9179

DOI: http://dx.doi.org/10.33238/ReBECEM.2019.v.3.n.3.23595

Desse modo, percebemos a partir dos estudos de Miguel e Miorim (2002) em relação aos trabalhos que aqui apresentamos, a dimensão do campo investigativo em História da Matemática, que por sua vez não se limita a um aspecto trivial, mas que dimensiona na prática social investigativa a história de acordo com as necessidades humanas em conformidade a D'Ambrosio (1999), que tem servido para revelar a história de práticas humanas e/ou práticas pedagógicas, história de conteúdos e saberes, história de instituições que envolvem currículo, história que envolve pessoas, memórias, documentos, mas sobretudo, pesquisas históricas que se preocupam e tem como essência revelar fragmentos do complexo campo da História da Matemática.

\section{Referências}

ARAUJO NETO, A. P. Um estudo histórico do curso de matemática da Universidade Estadual de Maringá: a criação e os primeiros anos. 2016. Dissertação (Mestrado em Educação para a Ciência e a Matemática) - Universidade Estadual de Maringá, Maringá, 2016.

BOGDAN, R. C.; BIKLEN, S. K. Investigação qualitativa em educação: uma introdução à teoria e aos métodos. Tradução de Maria J. Alvarez; Sara B. dos Santos; Telmo M. Baptista. Porto: Porto Editora, 1994.

BORTOLI, A. História da criação do Curso de Matemática na Pontifícia Universidade Católica de Campinas. 2003. Dissertação (Mestrado em Educação Matemática) - Universidade Estadual Paulista Júlio de Mesquita Filho, São Paulo, 2003.

CERVO, A. L.; BERVIAN, P. A.; SILVA, R. Metodologia científica. 6. ed. São Paulo: Pearson, 2007.

CASTRO E ALMEIDA, S. P. N. Um lugar: muitas histórias o processo de formação de professores de Matemática na primeira instituição de ensino superior da região de Montes Claros/norte de Minas Gerais (1960-1990). 2015. Tese (Doutorado) - Universidade Federal de Minas Gerais, Faculdade de Educação, Belo Horizonte, 2015.

D’AMBROSIO, U. A História da Matemática: questões historiográficas e políticas e reflexos na Educação Matemática. In: BICUDO, M. A. V. (org.). Pesquisa em Educação Matemática: concepções e perspectivas. São Paulo: UNESP, 1999, p. 97-115.

GARNICA, A. V. M.; SOUZA, L. A. Elementos de História da Educação Matemática. São Paulo: Cultura Acadêmica, 2012.

LIMA, G. L. A disciplina de Cálculo I do curso de Matemática da Universidade de São

Paulo: um estudo de seu desenvolvimento, de 1934 a 1994. 2012. Tese (Doutorado em Educação Matemática) - Pontifícia Universidade Católica de São Paulo, São Paulo, 2012.

MACHADO, S. R. A. As modificações curriculares do Curso de Matemática da Universidade Estadual de Maringá: mudanças no saber profissional do professor de matemática, 1971-1996. 2019. Dissertação (Mestrado em Educação para a Ciência e a Matemática) - Universidade Estadual de Maringá, Maringá, 2019. 
DOI: http://dx.doi.org/10.33238/ReBECEM.2019.v.3.n.3.23595

MACHADO, S. R. A.; TRIVIZOLI, L. M. Uma história para o inventário arquivístico do Departamento de Matemática da UEM. Boletim Cearense de Educação e História da Matemática, Fortaleza, v. 6, p. 56-70, 2019.

MENDES, I. A. Pesquisas em história da Educação Matemática no Brasil em três dimensões. Quipu, México, v. 14, n. 1, p. 69-92, jan. 2012.

MIGUEL, A.; MIORIM, M. Â. História da Matemática: uma prática social de investigação em construção. Educação em Revista, Belo Horizonte, n. 36, p. 161-176, 2002.

MIORIM, M. Â.; MIGUEL, A. A constituição de três campos afins de investigação: história da Matemática, educação matemática e história \& educação matemática. Revista Teoria e Prática da Educação, Maringá, v. 4, n. 8, p. 35-62, 2001.

PIZZANI, L. et al. A arte da pesquisa bibliográfica na busca do conhecimento. RDBCI: Revista Digital de Biblioteconomia e Ciência da Informação, Campinas, v. 10, n. 1, p. 53-66, 2012.

SAD, L. A. Comunidade científica de História da Matemática: uma trajetória de sua difusão e de eventos produtores. In: SEMINÁRIO NACIONAL DE HISTÓRIA DA MATEMÁTICA, 4. 2005, Brasília. Anais... Rio Claro, SP: Sociedade Brasileira de História da Matemática, 2005. p. $1-6$.

SANTOS, M. G. dos. Formação superior específica de professores de matemática no Espírito Santo: uma história de 1964 a 2000. 2013. Dissertação (Mestrado em Educação) Universidade Federal do Espírito Santo, Vitória, 2013.

SOARES, S. R. Um estudo histórico do ensino de geometria analítica no curso de matemática da UFJF nas décadas de 1960 e 1970. 2013. Dissertação (Mestrado Profissional em Educação Matemática) - Universidade Federal de Juiz de Fora, Juiz de Fora, 2013.

TRIVIZOLI, L. M. Sinais da organização da comunidade matemática brasileira: Sociedade de Matemática de São Paulo. Revista Brasileira de História da Matemática, Rio Claro. v. 9, p. 153-168, 2009.

TRIVIZOLI, L. M. Um panorama para a investigação em História da Matemática: surgimento, institucionalização, pesquisa e métodos. Revista Paranaense de Educação Matemática, Campo Mourão, v. 5, p. 189-212, 2016.

VALGAS, C. L. Licenciatura em Matemática: aspectos históricos e curriculares da UEPG. 2002. Dissertação (Mestrado em Educação) - Universidade Estadual de Ponta Grossa, Ponta Grossa, 2002.

ZICCARDI, L. R. N. O curso de Matemática da PUC/SP: uma história de sua construção/desenvolvimento/legitimação. 2009. Tese (Doutorado em Educação) - Pontifícia Universidade Católica de São Paulo, São Paulo, 2009.

Recebido em: 15 de novembro de 2019. Aceito em: 07 de dezembro de ano 